\title{
Contribution of outgrowth endothelial cells from human peripheral blood on in vivo vascularization of bone tissue engineered constructs based on starch polycaprolactone scaffolds ${ }^{i / 5}$
}

\author{
Sabine Fuchs ${ }^{\mathrm{a}, *, 1}$, Shahram Ghanaati ${ }^{\mathrm{a}, 1}$, Carina Orth ${ }^{\mathrm{a}}$, Mike Barbeck ${ }^{\mathrm{a}}$, Marlen Kolbe ${ }^{\mathrm{a}}$, \\ Alexander Hofmann ${ }^{\mathrm{b}}$, Markus Eblenkamp ${ }^{\mathrm{c}}$, Manuela Gomes ${ }^{\mathrm{d}}$, Rui L. Reis ${ }^{\mathrm{d}}$, Charles J. Kirkpatrick ${ }^{\mathrm{a}}$ \\ ${ }^{a}$ Institute of Pathology, Langenbeckstr. 1, Johannes Gutenberg University, 55101 Mainz, Germany \\ ${ }^{\mathrm{b}}$ Department of Trauma Surgery, Johannes Gutenberg University, Mainz, Germany \\ ${ }^{\mathrm{c}}$ Department of Medical Engineering, Technical University, Munich, Germany \\ d 3B's Research Group - Biomaterials, Biodegradables and Biomimetics, IBB - Institute for Biotechnology and Bioengineering, \\ PT Government Associated Laboratory, University of Minho, Campus de Gualtar, Braga, Portugal
}

\section{A R T I C L E I N F O}

\section{Article history:}

Received 4 August 2008

Accepted 23 September 2008

Available online 31 October 2008

\section{Keywords:}

Endothelial progenitor cells

Vascularization

Bone tissue engineering

In vivo test

Osteoblasts

\begin{abstract}
A B S T R A C T
In the present study we assessed the potential of human outgrowth endothelial cells (OEC), a subpopulation within endothelial progenitor cell cultures, to support the vascularization of a complex tissue engineered construct for bone. OEC cultured on starch polycaprolactone fiber meshes (SPCL) in monoculture retained their endothelial functionality and responded to angiogenic stimulation by VEGF (vascular endothelial growth factor) in fibrin gel-assays in vitro. Co-culture of OEC with human primary osteoblasts (pOB) on SPCL, induced an angiogenic activation of OEC towards microvessel-like structures achieved without additional supplementation with angiogenic growth factors. Effects of co-cultures with $\mathrm{pOB}$ on the vascularization process by OEC in vivo were tested by subcutaneous implantation of Matrige $\mathrm{I}^{\circledR}$ plugs containing both, OEC and pOB, and resulted in OEC-derived blood vessels integrated into the host tissue and anastomosed to the vascular supply. In addition, morphometric analysis of the vascularization process by OEC indicated a better performance of OEC in the co-cultures with primary osteoblasts compared to monocultures of OEC. The contribution of OEC to vascular structures and the beneficial effect of the co-culture with primary human osteoblasts on the vascularization in vivo was additionally proven by subcutaneous implantation of pre-cellularized and pre-cultured SPCL constructs. OEC contributed to the vascular structures, by generating autogenic vessels or by incorporation into chimeric vessels consisting of both, human and mouse endothelial cells. The current data highlight the vasculogenic potential of OEC for bone tissue engineering applications and indicate a beneficial influence of constructs including both osteoblasts and endothelial cells for vascularization strategies.
\end{abstract}

(c) 2008 Elsevier Ltd. All rights reserved.

\section{Introduction}

Therapeutic success in tissue engineering and regenerative medicine is highly dependent on an appropriate vascularization of the bioengineered tissue. In this context, different strategies have been developed, including delivery systems for angiogenic growth factors embedded in the biomaterials, as well as the generation of prevascularized tissues by incorporation of endothelial cells. The latter might facilitate an anastomosis of bioengineered vascular

\footnotetext{
is This work was performed at the Institute of Pathology in Mainz, Germany.

* Corresponding author. Tel.: +49 613117 4204; fax: +496131 175645.

E-mail address: fuchss@uni-mainz.de (S. Fuchs).

1 Both authors are contributed equally.
}

structures with those in the peri-implant tissue, as has been recently shown in a prevascularized tissue engineered construct for muscle [1]. From several studies we have learned that a variety of cell types such as fibroblasts [2], smooth muscle cells [3] adipocytes [4] or osteoblasts $[5,6]$ etc. can support the generation of prevascular structures by mechanisms which are currently under further investigation. The cellular cross talk between endothelial cells and other cell types comprises paracrine mechanisms based on several growth factors such as VEGF and PDGF [7], as well as direct cell to cell communication [7,8]. These molecular and cellular processes seem to control the formation of vascular structures in a close relation to the physiological conditions such as hypoxia [9].

Beyond the research interest in the molecular and cellular regulation of angiogenesis and vasculogenesis, the concept of co-cultures has also influenced research directed towards new therapeutic 
approaches for critical bone defects in tissue engineering. Recent findings regarding the mutual information exchange between osteoblasts and endothelial cells or their corresponding precursors, respectively, have led to marked interest in the generation of more complex and appropriately vascularized tissue engineered products for bone tissue [10-13].

Nevertheless, for such "pre-endothelialized" or "prevascularized" tissue constructs autologous cell sources are an important prerequisite, before they may be considered for a clinical application. Therefore, endothelial progenitor cells become of interest as a potential autologous cell source for endothelial cells. In particular, one subpopulation within EPC, so called outgrowth endothelial cells (OEC) or late endothelial progenitor cells [14-16] with endothelial characteristics with respect to phenotype, as well as on the functional level, seem to meet the requirements for a therapeutic application. OEC can be isolated from the peripheral blood as an easily obtainable source from an adult patient. Furthermore, OECs are a homogenous and distinct population of cells revealing the potential for in vitro expansion as well as a stable endothelial phenotype [17]. Furthermore, OEC contribute to the neovascularization after implantation in vivo $[16,18]$. Nevertheless, in vivo studies also revealed that stabilizing cells such as smooth muscle cells are needed to stabilize vascular structures formed in long term in vivo experiments [19].

In our previous in vitro studies OEC formed prevascular structures with a vascular lumen in co-culture with osteoblastic cells lines or primary osteoblasts, indicating a beneficial effect of this co-culture technique on the angiogenic activation of OEC [6]. Nevertheless, in bone tissue engineering biomaterials are used to fill bone defects to ensure mechanical stability and serve as scaffolding material for the regenerating tissue and therapeutical cells. In previous studies fiber mesh scaffolds generated from a blend of starch with polycaprolactone (SPCL) have been shown to support both endothelial cells [20] and growth of bone marrow cells [21,22]. In the present study, we evaluated the in vitro biofunctionality of OEC on SPCL fiber meshes in terms of their endothelial phenotype, as well as the angiogenic activation in response to angiogenic stimuli in vitro. In addition, we generated more complex cellular constructs based on SPCL fiber meshes consisting of both human OEC and primary human osteoblast in a co-culture system. Finally, we assessed the formation of vascular structures by OEC in vivo and the impact of co-cultures with pOB on the vascularization process in subcutaneous implantation models based on Matrige ${ }^{\circledR}$ plugs and complex SPCL-based constructs.

\section{Materials and methods}

\subsection{Isolation of human outgrowth endothelial cells}

Outgrowth endothelial cells (OEC) were isolated according to previous published protocols $[6,17,23]$. In brief, mononuclear cells were isolated from human peripheral blood buffy coats by ficoll- (Sigma-Aldrich, Steinbach, Germany) gradient centrifugation and cultured in EGM-2 (endothelial cell growth medium-2, CC-3162, Lonza, Verviers, Belgium). Culture medium was supplemented with the kit ingredients, $5 \%$ fetal calf serum (FCS, GIBCO Life Technologies, Karlsruhe, Germany) and $1 \%$ Pen/Strep. $5 \times 10^{6}$ cells/well were seeded on a 24 culture-well plate coated with collagen (BD Europe, Heidelberg, Germany). Colonies of outgrowth endothelial cells with endothelial morphology appearing after 3-4 weeks were trypsinized and expanded during several passages in a splitting ratio $1: 2$ or $1: 3$. The purity and homogeneity of OEC (about 95\%) was determined by flow cytometry. HUVEC which were used as controls were isolated and cultured as previous described.

\subsection{Culture of human osteoblasts}

Endosteal cancellous bone fragments from hip or knee were derived from 4 female or male patients (age 60-81) undergoing orthopaedic and trauma surgery, who had given their consent for material use. Investigations were approved by the local ethical committee. Primary osteoblasts were isolated and cultured according to an outgrowth protocol as previously described [24]. In brief, bone fragments were minced and rinsed in PBS (phosphate buffered saline) for several times followed digestion of the bone tissue by collagenase (Type IV C-5138, Sigma, Deisenhofen, Germany) for one hour at $37^{\circ} \mathrm{C}$. After this digestion step, bone fragments were rinsed again with PBS for several times. Bone fragments were incubated in DMEM/ Hams F12 (GIBCO) including 20\% FCS and 1\% Pen/Strep to allow the outgrowth of the cells from the bone fragments. Confluent cultures were passaged using acutase (PAA) in a ratio of 1:3 and further cultured in DMEM/Hams F12 supplemented with $10 \%$ FCS. For the present studies cells passage 1 to passage 4 were used to generate co-cultures.

\subsection{Generation of SPCL constructs}

SPCL scaffolds were generated and sterilized as previously described [22]. Prior to cell seeding scaffolds were coated with fibronectin according to standard protocols [20]. SPCL were seeded with 500.000 OEC per scaffold with a base of $0.33 \mathrm{~cm}^{2}$ or, respectively, 250.000 primary osteoblasts and 250.000 OEC for the cocultures. The same cell culture medium, EGM-2 with $5 \%$ FCS; was used in both set ups, monocultures or co-cultures according to the previously established protocols [6] without additional supplementation with angiogenic growth factors if not stated otherwise.

\subsection{Scanning electron microscopy of outgrowth endothelial cells on SPCL}

Samples of SPCL seeded with OEC for scanning electron microscopy (SEM) were treated as previously described [23] using standard procedures. In brief, samples were fixed with $2 \%$ glutaraldehyde in $0.1 \mathrm{~m}$ cacodylate buffer for $30 \mathrm{~min}$. Samples were treated with $1 \%$ osmium tetroxide for $1 \mathrm{~h}$, followed by dehydration using increasing concentrations of acetone. Finally, samples were critically point dried and sputtered with gold before analysis.

\subsection{Immunofluorescent staining of cellular constructs}

Outgrowth endothelial cells alone or in co-culture with osteoblastic cells on SPCL were fixed using $2 \%$ paraformaldehyde (PFA). After washing with PBS cells were permeabilized by $0.1 \%$ Triton- $X$, followed by the incubation with primary antibodies, anti-human von Willebrand (vWF) (A0082 Dako, Hamburg, Germany, diluted 1:8000), anti-human CD31 (M0823Dako, Hamburg, Germany, diluted 1:50), VE-Cadherin (C-26120 Transduction, Lexington, UK, diluted $1: 100)$, in $1 \%$ bovine serum albumin (BSA)/PBS for $40 \mathrm{~min}$ at room temperature. Then cells were washed and labeled with the corresponding Alexa anti-mouse or antirabbit conjugates, Alexa 488 anti-rabbit (A-21206), Alexa 488 anti-mouse (A11029), Alexa 546 anti-rabbit (A-11010), Alexa 546 anti-mouse (A-11030) (all from Molecular Probes) diluted $1: 1000$ in $1 \%$ BSA/PBS for $40 \mathrm{~min}$ at room temperature. Nuclear counterstaining was routinely performed using Hoechst or PI (propidium iodide), respectively. Finally constructs were mounted with Gelmount (M01, Biomeda, Foster City, USA) and studied by confocal microscopy using a LeicaTCS-NT.

\subsection{Gene expression in monoculture of OEC or in co-cultures on SPCL}

Total RNA was extracted from OEC grown for 1 week on SPCL scaffolds using RNeasy Kit (Qiagen, Hilden, Germany). From the co-cultures RNA was extracted after two different time points after 1 and after 4 weeks of co-culturing. RNA isolation was performed including the treatment of isolated RNA with DNAse according to standard protocols to prevent impurities by genomic DNA. RNA was extracted directly from the cells grown on the scaffolds by adding RNA-later buffer. $1 \mu \mathrm{g}$ of RNA was then transcribed into cDNA according to protocols from the Omniscript RT kit (Qiagen).

\subsection{Relative quantification (RQ) of gene expression by real time $P C R$}

Quantitative real time PCR was performed with the use of Applied Biosystems 7300 Real Time PCR System (Applera Deutschland GmbH, Darmstadt, Germany). The reaction composition was the following: $12.5 \mu \mathrm{l}$ of $2 \times$ QuantiTect $^{\mathrm{TM}}$ SYBR $^{\circledR}$ Green PCR Master Mix, $2.5 \mu$ of $10 \times$ QuantiTect $^{\mathrm{TM}}$ SYBR $^{\circledR}$ Green Primer Assay (see Table 1), $5 \mu \mathrm{l}$ of RNase-free water and $5 \mu \mathrm{l}$ of cDNA pre-diluted 1:100 with RNase-free water. All the reagents were provided by QIAGEN GmbH (Hilden, Germany). Each gene was processed in triplicate. The specificity of PCR was assessed in a preliminary step on the basis of dissociation curves. The relative quantification of gene expression was assessed using the Applied Biosystems Sequence Detection Software v.1.2.2. The housekeeping gene GAPDH was found to have the most consistent expression level and was therefore chosen as an endogenous control for the experiments. The ratio between the gene expression levels in OEC grown on SPCL versus tissue culture plastic or in co-cultures of 1 or 4 weeks, respectively, was determined as indicated in the individual graphs and the difference was considered to be significant, if $p$-values obtained from the paired $t$-test were less than 0.05 .

\subsection{D-fibrin angiogenesis assays}

OEC in monoculture were grown on the SPCL scaffolds for 1 week as described above. To assess the response of OEC towards angiogenic stimulation 
Table 1

QuantiTect ${ }^{\mathrm{TM}}$ SYBR $^{\circledR}$ Green Primer Assays used in the experiment

\begin{tabular}{lll}
\hline Gene name & Primer Assay name & Catalogue number \\
\hline PECAM & Hs_PECAM1_1_SG QuantiTect & QT00081172 \\
VE-Cadherin & $\begin{array}{l}\text { Primer Assay } \\
\text { Hs_CDH5_1_SG QuantiTect }\end{array}$ & QT00013244 \\
Primer Assay & Hs_VWF_1_SG QuantiTect & QT00051975 \\
Alkaline phosphatase & $\begin{array}{l}\text { Primer Assay } \\
\text { Hs_ALPL1_SG QuantiTect }\end{array}$ & QT00012957 \\
Osteocalcin & $\begin{array}{l}\text { Primer Assay } \\
\text { Hs_BGLAP_1_SG QuantiTect }\end{array}$ & QT00232771 \\
GAPDH & $\begin{array}{l}\text { Primer Assay } \\
\text { Hs_GAPDH_1_SG QuantiTect }\end{array}$ & QT00079247 \\
& Primer Assay & \\
\hline
\end{tabular}

constructs were embedded in fibrin gels as previously described [23] using the in vitro Angiogenesis Assay Kit (ECM 630, Chemicon). After gelation fibrin gels were covered with $1 \mathrm{ml}$ of EGM-2 containing $10 \mathrm{ng} / \mathrm{ml}$ VEGF and the medium was changed every second day. After 1 week the fibrin gels were fixed and further processed for immunofluorescent staining as described in the previous section.

\subsection{Subcutaneous implantation of Matrigel ${ }^{\circledR}$ plugs and in vivo assessment of} cellular constructs of SPCL

This experiment was approved by the Committee on the Use of Live Animals in Teaching and Research, Rheinland-Pfalz, Germany. Six male 6-week-old SCID mice (Charles River Laboratories, Germany) were bred and kept under standard conditions and provided with water ad libitum, artificial light and normal mouse pellet (Laboratory Rodent Chow, Altromin, Germany) at the Laboratory Animal Unit, Johannes Gutenberg University, Mainz, Germany. For Matrigel ${ }^{\circledR}$ plug experiments OEC from 3 different donors were trypsinized and $3 \times 10^{6}$ OEC were resuspended in $400 \mu \mathrm{l}$ Matrigel ${ }^{\circledR}$. For the co-implantation with pOB $1.5 \times 10^{6}$ and $1.5 \times 10^{6}$ OEC were

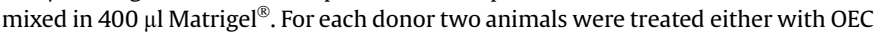
Matrige ${ }^{\circledR}$ or OEC/pOB -Matrigel ${ }^{\circledR}$ suspension. Samples were kept on ice before the cell containing Matrigel ${ }^{\circledR}$ was injected using a 20-gauge needle percutaneosly into the subcutaneous region of the nude mice.

For the generation of cellular constructs SPCL were seeded either with OEC alone ( 500.000 cells per scaffold), or both cell types (pOB, OEC; 250.000 cells each). The cellular constructs were prepared and pre-cultured for 1 week as previously described. For some experiments cellular constructs on SPCL were embedded in 300-400 $\mu$ Matrigel $^{\circledR}$, before implantation and after gelation the constructs were implanted as described below.

Eight male 6-week-old SCID mice (Charles River Laboratories, Germany) were bred and kept under standard conditions and provided with water ad libitum, artificial light and normal mouse pellet (Laboratory Rodent Chow, Altromin, Germany). In vitro generated constructs were subcutaneously implanted in preformed subcutaneous pockets of the subscapular region of the nude mice according to the modified method of Hafemann [25]. The experimental group consisted of four animals which were treated with SPCL + OEC from donor A or SPCL + OEC + pOB from donor $\mathrm{A}, \mathrm{SPCL}+\mathrm{OEC}$ from donor $\mathrm{B}$ or $\mathrm{SPCL}+\mathrm{OEC}+\mathrm{pOB}$ from donor B. All SPCL scaffolds of this group were embedded in Matrigel ${ }^{\circledR}$ (BD Biosciences, USA). Two animals served as control for the the effects of Matrigel ${ }^{\circledR}$ and were implanted with SPCL + OEC + pOB from either donor A or donor B, but these SPCL scaffolds were not embedded in Matrigel ${ }^{\circledR}$. As quality assurance measures for the in vivo experiments as well as for the histomorphometrical analysis, one animal was treated with SPCL embedded in Matrigel ${ }^{\circledR}$ (BD Biosciences, USA) and one animal underwent a sham operation (i.e., a mock operation without biomaterial implantation).

\subsection{Histology and immunohistochemistry}

After 14 days, mice were euthanized with an overdose of ketamine and xylazine. Biomaterials were explanted together with the surrounding peri-implant tissue, fixed in $4 \%$ formalin for $24 \mathrm{~h}$, embedded in paraffin and prepared for histological staining. Sections of $3 \mu \mathrm{m}$ thickness were cut from the explanted tissue and stained with Mayer's haematoxylin and eosin (HE-staining). Von Willebrand Factor antibodies (Dako, Sweden) detecting specifically human endothelial cells or isolectin B4 (Vector Laboratories, Burlingame, CA) used as a murine endothelial cell marker were visualized with diaminobenzidine (Vector Laboratories). For negative controls, sections were treated similarly but omitting the primary antibody. Histopathological evaluation was performed using a Nikon ECLIPSE 80i microscope (Nikon, Japan) by two independent examiners blinded to the experimental protocol. Microphotographs were taken using a Nikon DS-Fi1/Digital camera and a digital sight control unit (Nikon, Japan) and prepared for further histomorphological analysis.

\subsection{Histomorphometrical analysis}

All images showing human cells (immunopositive for von Willebrand Factor) were subjected to automatic quantification of the human cells using the S.CORE Image Analysis System (S.CO LifeScience, Germany): In a first step all brown immunopositive structures were separated from the background with a thresholdbased approach. Then the brown elements were separated into areas with a weakdiffuse staining and those with an intensive-condensed staining. All objects with a weak-diffuse staining as well as very small objects with an intensive-condensed staining were classified as 'unspecific staining'. All other objects (bigger objects with intensive-condensed staining) were classified as 'specific staining'. The total area of all objects with specific staining was determined as the parameter for the content of human cells within the histological sections.

\section{Results}

\subsection{Endothelial phenotype of outgrowth endothelial cells on SPCL}

Outgrowth endothelial cells in monoculture adhered to the single fibres of the SPCL scaffold material and formed an endothelial cell layer. OEC seemed to retain the typical endothelial phenotype on the SPCL fiber meshes which was demonstrated by several methods such as scanning electron microscopy (Fig. 1A and B). Immunofluorescent staining for endothelial markers such as CD31 (Fig. 1D), VE-cadherin and vWF (data not shown) revealed the formation of an interconnected endothelial cell layer. Cellular contact molecules CD31 and VE-cadherin were situated along the cellular contacts, comparable to HUVECs used as endothelial controls (Fig. 1C). The morphology of the endothelial cell layers was similar to controls which were run on tissue culture plastic. Similar findings with regard to endothelial cells were observed using additional methods investigating the expression of endothelial cell markers such as CD31, VE-cadherin and vWF by Real Time PCR (Fig. 1E). Relative gene expression of endothelial markers was compared in cells grown on plastic (reference values set to 1 ) and cells grown on the SPCL scaffold material by quantitative Real Time PCR. The expression of CD31 and vWF was significantly reduced in contrast to VE-cadherin, for which we only observed a slight decrease with no statistical significance.

\subsection{Response of outgrowth endothelial cells to angiogenic stimulation}

To assess the ability of OEC to respond to angiogenic stimuli and to form microvessel-like structures OEC grown for 1 week on the SPCL were embedded in a fibrin gel and the growth factor VEGF was added to the cell culture medium as an angiogenic stimulus. By immunostaining for the endothelial marker CD31 (depicted in green, nuclear counterstaining in red) and confocal laser scanning microscopy (Fig. 2A and B) we observed the angiogenic activation of outgrowth endothelial cells leading to angiogenic sprout formation.

The angiogenic activation of OEC in more complex tissue engineered constructs based on co-cultures with human primary osteoblasts on SPCL fiber meshes was first assessed in vitro. Using confocal microscopy co-cultures of OEC and pOB were investigated after 1 week of co-culturing (Fig. 2C and D). OEC were identified by staining for the endothelial marker CD31, depicted by green fluorescence. Nuclear counterstaining was used to detect all cells, including primary osteoblasts. OEC were embedded in a rich matrix produced by primary osteoblasts and showed an elongated morphology and an organization into microvessel-like structures indicating an angiogenic activation by the co-culture process. The maturity of those vascular sprouts increased with culture time (4 weeks, Fig. 2E and F). Nevertheless, as the formation of vascular structures was already initiated after 1 week of co-culture, the cellular constructs based on SPCL were implanted after 1 week of pre-culture for further in vivo evaluation in terms of the angiogenic potential of OEC. 

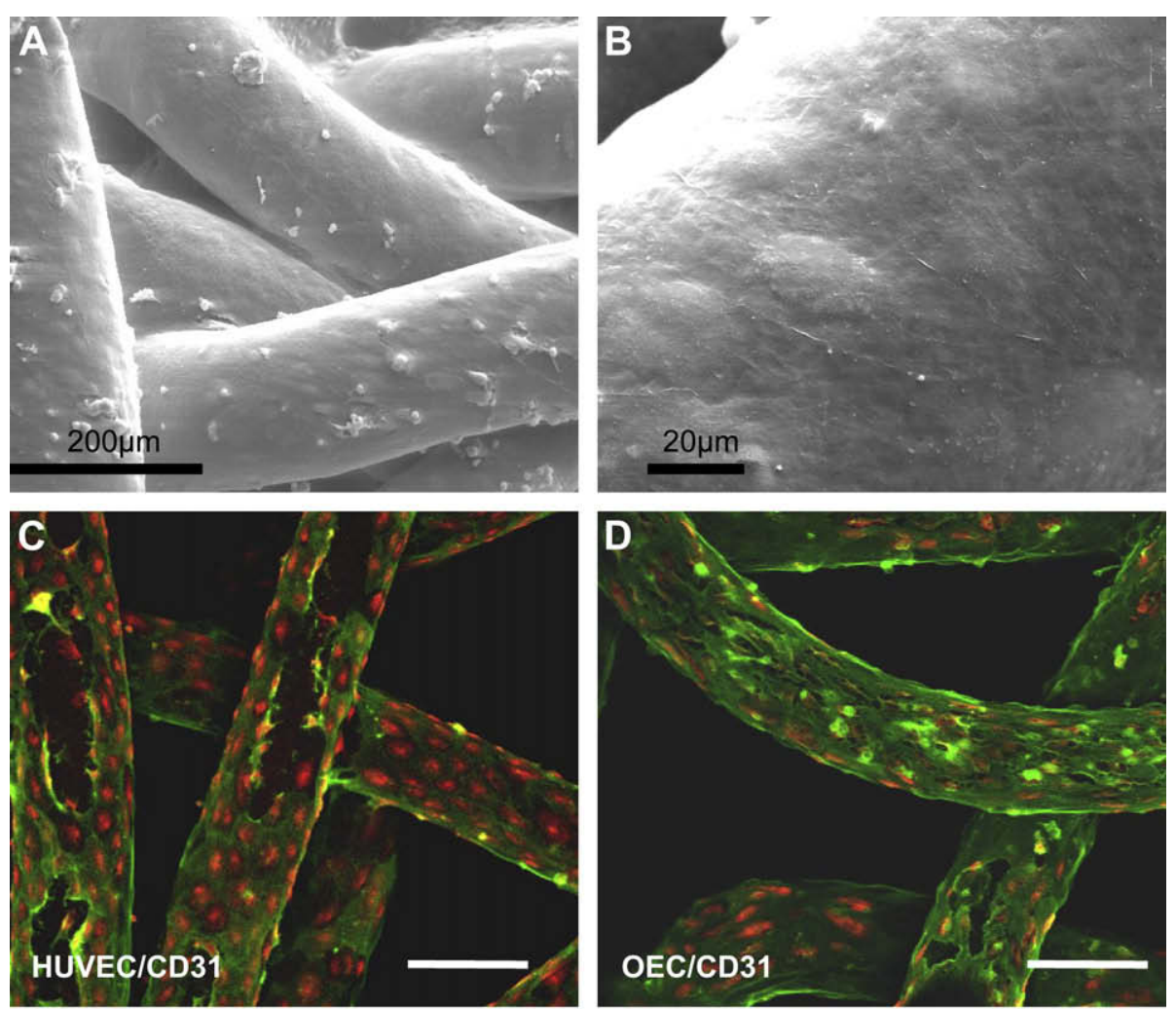

$\mathbf{E}$

GAPDH endocontrol

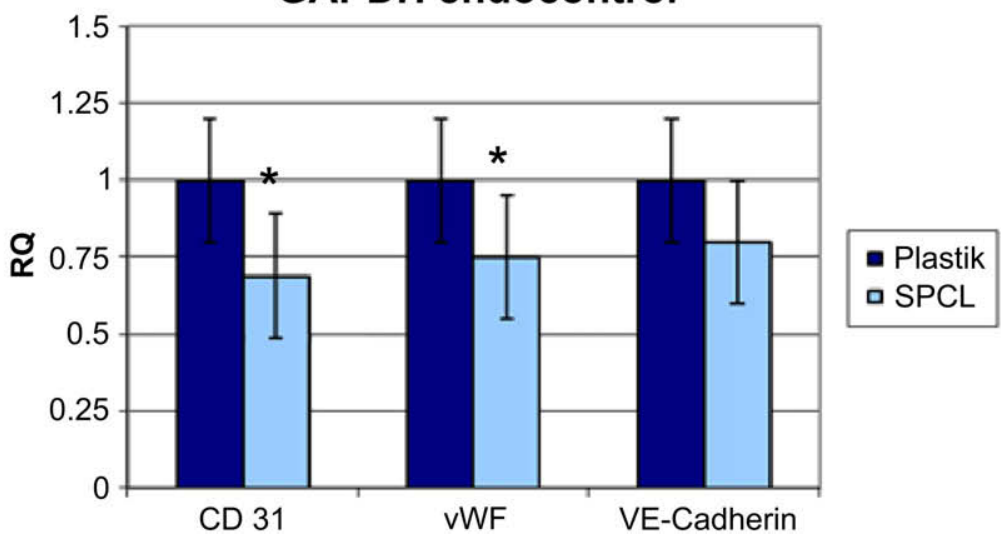

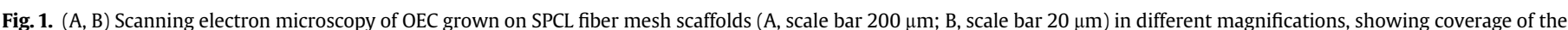

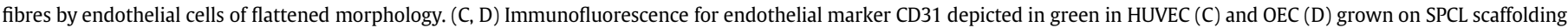

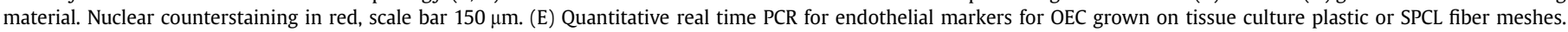

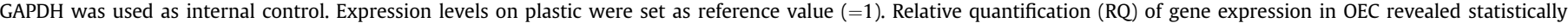
significant downregulation of CD31 and vWF but not of VE-cadherin. * Indicate statistical significance $\leq p$-value 0.05 .

\subsection{Osteogenic potential of SPCL constructs}

The potential of osteogenic differentiation of the SPCL constructs under the applied culture conditions was assessed by quantitative real time PCR for alkaline phosphatase and osteocalcin comparing the expression rates after 1 and 4 weeks in the coculture (Fig. 3). In the investigated time frame we observed a significant downregulation of alkaline phosphatase and a significant increase in the expression of osteocalcin. ( $p$-values $\leq 0.05$ ).

\subsection{Subcutaneous implantation of Matrigel plugs}

In order to evaluate the angiogenic potential of OEC from different donors in vivo and to assess the impact of the co-culture with primary osteoblasts on OEC-derived vascular structures, Matrigel ${ }^{\circledR}$ plugs containing either OEC alone or OEC and pOB were implanted subcutaneously (Fig. 4A-E). Perfused microvessels of mouse (Fig. 4A, ovals, von Willebrand factor-negative) or of human origin (Fig. 4A, arrows, von Willebrand factor-positive) with a diameter of up to $60 \mu \mathrm{m}$ were detected. In Fig. 4B-D the same area is depicted in stainings with differential specifity. The human origin of the vessels in this area was confirmed by a positive staining using human-specific van Willebrand antibodies (Fig. 4C) and the negative staining in a murine-specific isolectin staining (Fig. 4D). Morphometric analysis was used to quantify vascular structures comparing OEC implanted alone or co-implanted with pOB in Matrigel ${ }^{\circledR}$ plugs. For all tested donors $(n=3)$ the same trend was observed, indicating larger areas for human vascular structures in 

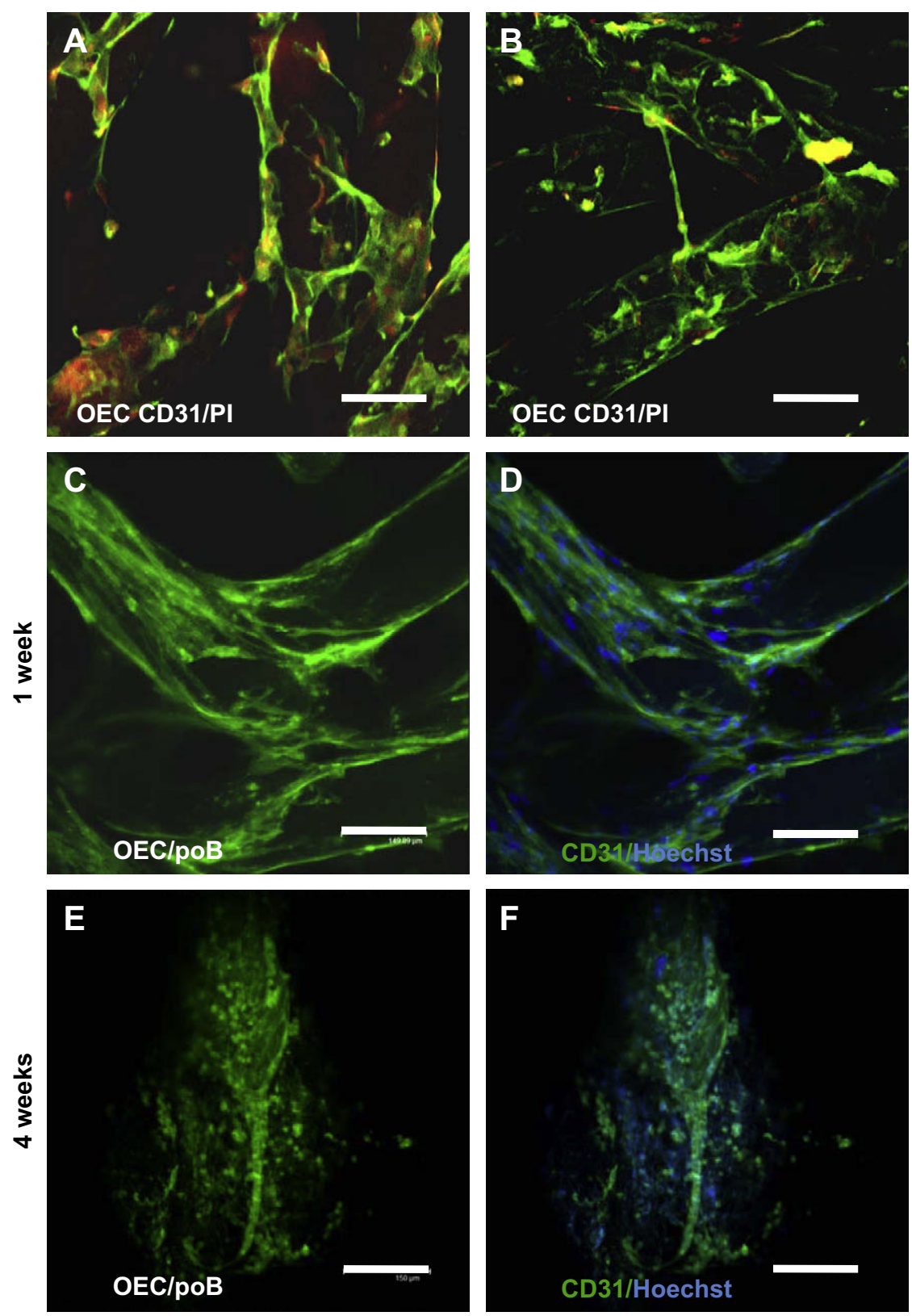

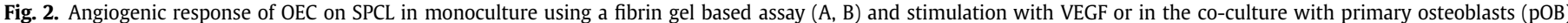

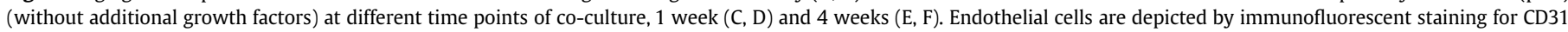

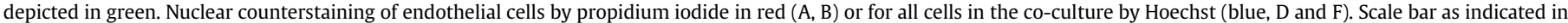
the picture $=150 \mu \mathrm{m}$. OEC show angiogenic activation using fibrin gels and supplementation with VEGF or in co-culture with primary osteoblasts.

co-implants of OEC and $\mathrm{POB}$ compared to controls using OEC alone (Fig. 4E).

\subsection{Vascularization of SPCL contructs in vivo containing OEC}

The SPCL fibres were well integrated into the surrounding periimplant tissue and were surrounded by both human (Fig. 5A; arrows) and murine microvessels (Fig. 5A; circles). Vascular structures in the peri-implant tissue were observed in both cases with (Fig. 5) or without using Matrigel ${ }^{\circledR}$ (not shown) as embedding matrix for the cellularized SPCL constructs. Nevertheless, embedding of constructs in Matrigel ${ }^{\circledR}$ seemed to have a beneficial effect on the formation of vascular structures in the peri-implant tissue (compare also Fig. 6). The contribution of OEC to the vascularization process was assessed using human-specific detection of vascular structures or detection of vascular structures formed by the host. Immunohistochemistry using the human-specific antibodies for vWF (Fig. 5A and B) revealed vascular structures formed by OEC mostly in close association with the SPCL fibres. These vascular structures formed by OEC revealed lumina (Fig. $5 \mathrm{~A}$ and $\mathrm{B}$ ) and contained erythrocytes as evidence of active perfusion. In control staining, detecting vascular structures formed by mouse, those vascular structures formed by OEC (Fig. 5B, red arrows) where negative for the mouse-specific staining (Fig. 5C, blue arrows). In addition, blood-filled chimeric vessels formed by both human and mouse endothelial cells were observed (Fig. 5D, highlighted by ovals).

Histomorphometrical analysis was performed in addition to assess the effect of Matrigel ${ }^{\circledR}$ as embedding material for the cellular constructs, as well as to gain insight into the effects of co-cultures 


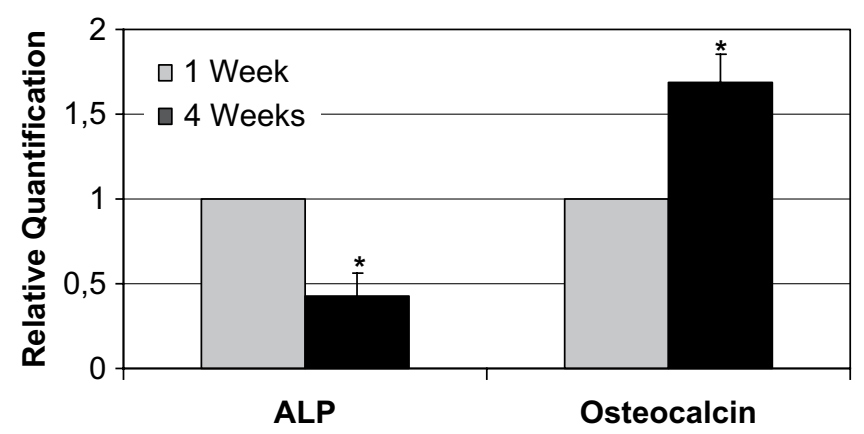

Fig. 3. Real time PCR for osteogenic markers alkaline phosphatase and osteocalcin in SPCL constructs consisting of endothelial cells and primary osteoblasts. Expression levels after 1 week of culture were set as reference value $(=1)$ and compared to 4 weeks of culture indicating a significant downregulation of alkaline phosphatase (ALP) and an increase in osetocalcin. ${ }^{*}$ Indicate statistical significance $\leq p$-value 0.05 . with pOB versus using OEC alone on the vascularization in the periimplant tissue (Fig. 6). Although there was a clear difference in the contribution of the different OEC donors to the vascularization of the SPCL constructs, embedding the cellular constructs in Matrigel ${ }^{\circledR}$ led to a clear increase of vascular structures in the peri-implant tissue. In addition, co-cultures consisting of OEC and primary osteoblasts showed a positive effect of the vascularization compared to the individual monocultures under the same experimental settings.

\section{Discussion}

In this study we present experimental evidence that OEC, a distinct population of cells with endothelial characteristics from the peripheral blood, can contribute to a functional vascularization in co-culture with $\mathrm{pOB}$ in a complex tissue engineered construct. These constructs consisted of primary human osteoblasts and OEC
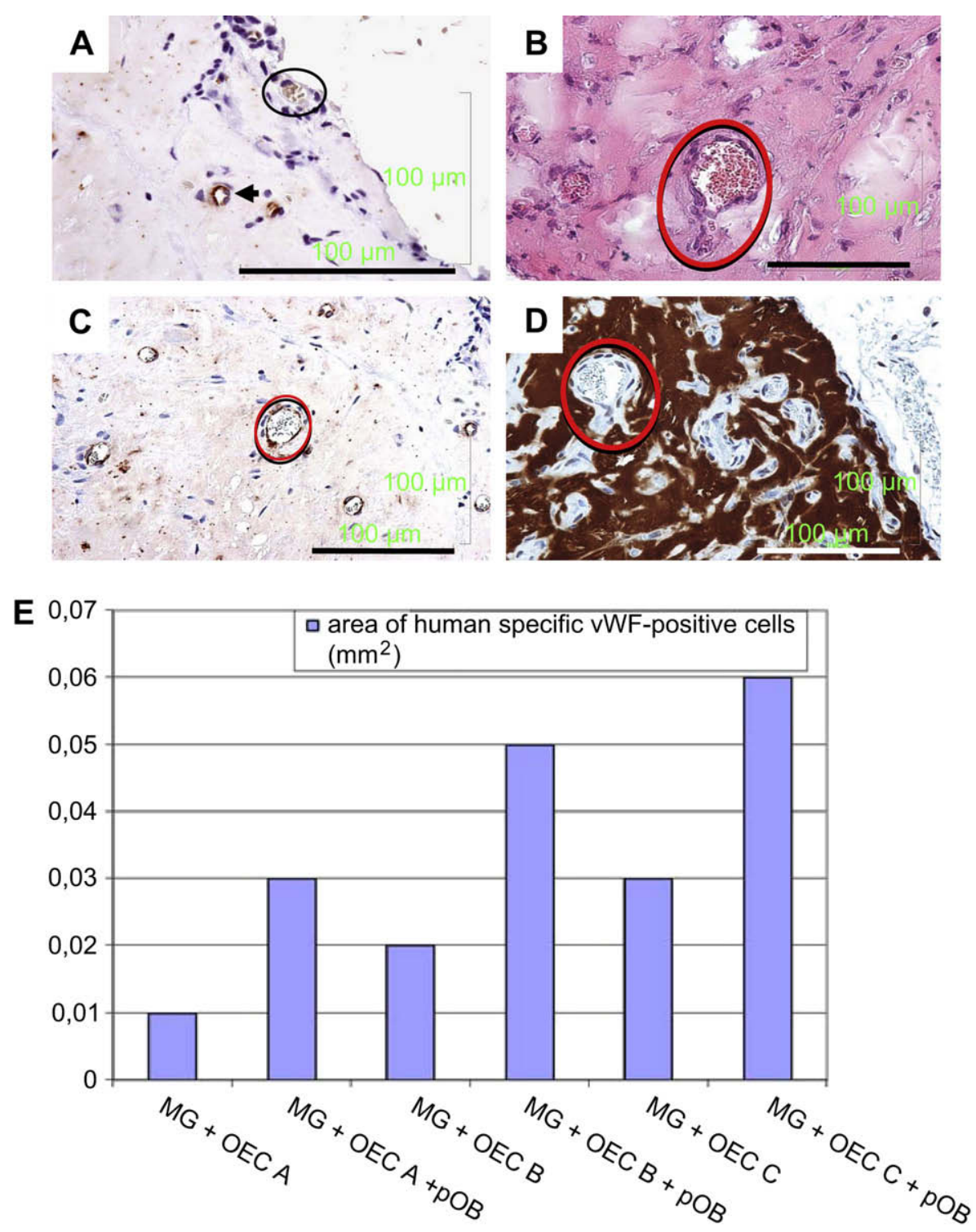

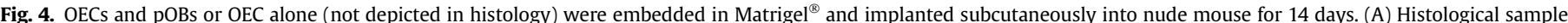

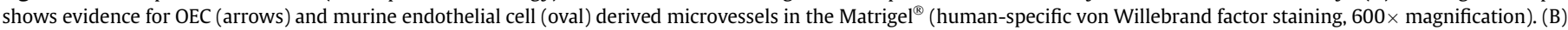

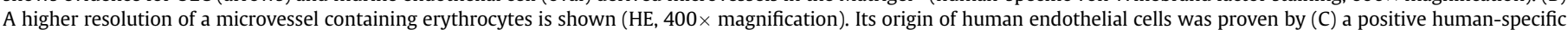

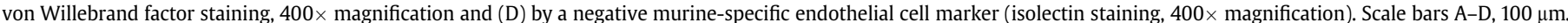

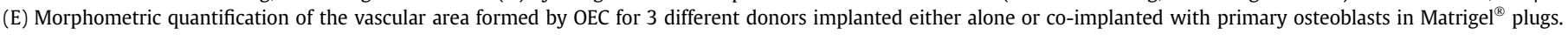



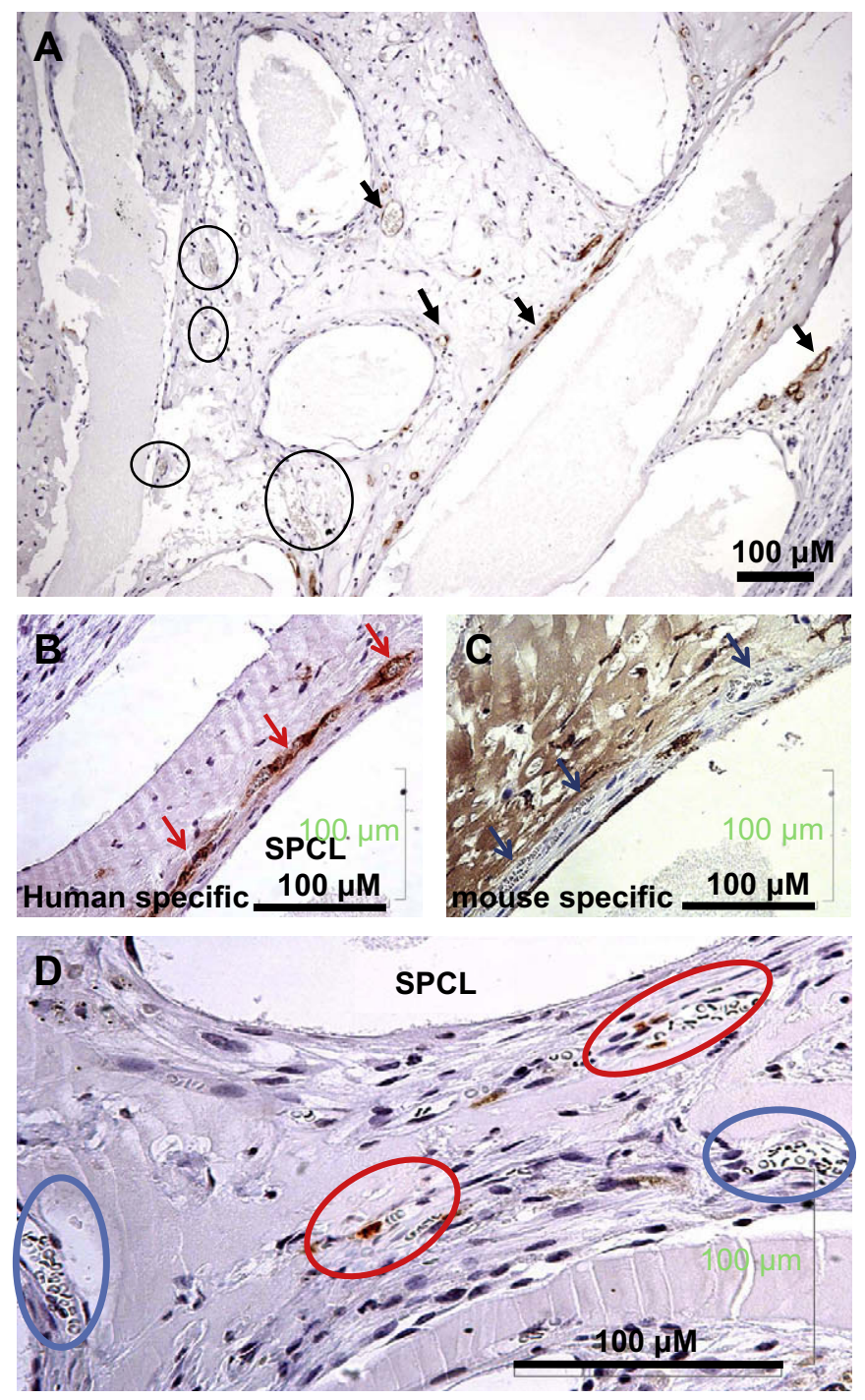

Fig. 5. The contribution of co-cultures consisting of pOB and OEC on SPCL embedded in Matrigel ${ }^{\circledR}$ to the vascularization of the peri-implant tissue of the nude mouse. (A) After implantation of cellular constructs, histological samples from the peri-implant tissue show evidence for contribution of OECs (arrows) and murine endothelial cells (ovals) to the formation of blood-filled vessels containing erythrocytes (humanspecific vWF-staining, Dako, Sweden, $100 \times$ magnification). (B) Human cell-derived microvessels both in cross and in longitudinal section (red arrows), (human-specific vWF-staining, Dako, Sweden, $400 \times$ magnification) remain unstained in their corresponding tissue segment after isolectin-B4 (C) staining for murine structures (blue arrows), (Isolectin B4 (Vector Laboratories, Burlingame, CA), 400× magnification). (D) In some vessels of the peri-implant tissue of nude mice treated with $\mathrm{OEC}+\mathrm{pOB}$ co-culture chimaeric vessels, containing both human and murine endothelial cells were observed (red ovals), (human-specific vWF-staining, Dako, Sweden, 400× magnification) pure murine vessels (blue ovals).

pre-cultured on SPCL fiber mesh scaffolds. Prevascular structures were induced by co-culturing OEC with primary osteoblasts on SPCL scaffolds, which were achieved without additional supplementation of culture medium with angiogenic growth factors. Co-implantation of OEC and pOB in Matrigel ${ }^{\circledR}$ plugs showed the contribution of OEC to perfused vascular structures and a beneficial effect on the vascular potential of OEC by co-implantation with pOB, although the reason for this observation deserves further investigation. Similar findings were also observed for co-cultures on SPCL. In cellular constructs consisting of OEC and primary osteoblasts on SPCL implanted subcutaneously into a nude mouse model, OEC formed vascular structures closely associated with the scaffold material and embedded in a rich extracellular matrix produced by the primary osteoblasts. Vascular structures formed by OEC were functionally connected to the vascular supply of the host as indicated by erythrocytes detected in luminal vascular structures. Additional experiments investigating the vascularization process in a time course experiment are currently under way to gain more insight into the dynamics of the vascularization process.

To some extent microvessel-like structures were already induced in vitro by a short pre-culturing phase in co-culture with primary osteoblasts. Such co-culture techniques seem to promote an angiogenic activation of OEC as indicated by our previous study inducing prevascular structures with definitive vascular lumina [6]. Similar findings were also observed for adult microvascular endothelial cells co-cultured with osteoblasts on silk fibroin scaffolds, where the organization of endothelial cells into vascular structures clearly increased with progressing culture time [5]. In the present study, morphometric quantification of the vessel area suggests that the co-culturing or co-implantation process with primary osteoblasts also has a beneficial influence on the formation of vascular structures in vivo. These observations are in accordance with several reports indicating a beneficial effect of cocultures consisting of endothelial cells with several other cell types for the vascularization process and the formation of durable blood vessels in vivo $[19,26]$. Several mechanisms such as embedding endothelial cells into a rich extracellular matrix or angiogenic activation of endothelial cells by paracrine factors are probably responsible for those beneficial effects. Furthermore, cocultures seem to support the stabilization of vessels by providing mural cells, which are essential, especially for the long term stabilization of newly formed blood vessels [16]. Although these studies have demonstrated the general potential of OEC to contribute to the vascularization process in vivo, these studies also emphasized that the therapeutical relevance of these cells critically depends on the experimental setting such as co-implantation with other cell types. Likewise, OEC revealed an altered vasculogenic potential in an endothelial spheroid-based implantation model in vivo compared to HUVEC (Finkenzeller et al., Cell Proliferation accepted) although the angiogenic activity of HUVEC and OEC in vitro were similar. Whether such differences in the vasculogenic potential in pure endothelial approaches derive from differences at the molecular level between mature or progenitor derived cells or from the macro- or microvascular character of HUVEC or OEC, respectively, still needs further investigation. Interestingly, recent studies comparing Affymetrix microarray data indicated that blood outgrowth endothelial cells were different from macrovascular cells (HUVEC), whereas they shared similarities with microvascular cells or may be classified as an individual subtype of the endothelial cell [27]. This is also in accordance with our observations from co-culture experiments with osteoblasts, comparing HUVEC and OEC [6].

Furthermore, embedding of cellular constructs based on SPCL into Matrigel ${ }^{\circledR}$ was highly supportive of the formation of vascular structures in the peri-implant tissue. Matrigel ${ }^{\circledR}$ might serve as a kind of stabilizing matrix for the cellular constructs and has been used in several studies to assess the angiogenic potential of OEC. Although the relevance of Matrigel ${ }^{\circledR}$ is limited in clinical applications, the present study suggests that embedding of cellular constructs into gels such as Matrigel ${ }^{\circledR}$ or hydrogels might be beneficial for the vascularization process of tissue engineered constructs. Moreover, on account of the possibility of biofunctionalization of those matrices, the tailored physico-chemical properties of such embedding matrices might be useful to control the migratory activity of the endothelial cells and to stabilize vascular structures.

The present study was specifically performed to answer the question of whether OEC could add to the vascularization of tissue engineered bone constructs based on 3D SPCL scaffolds and to 


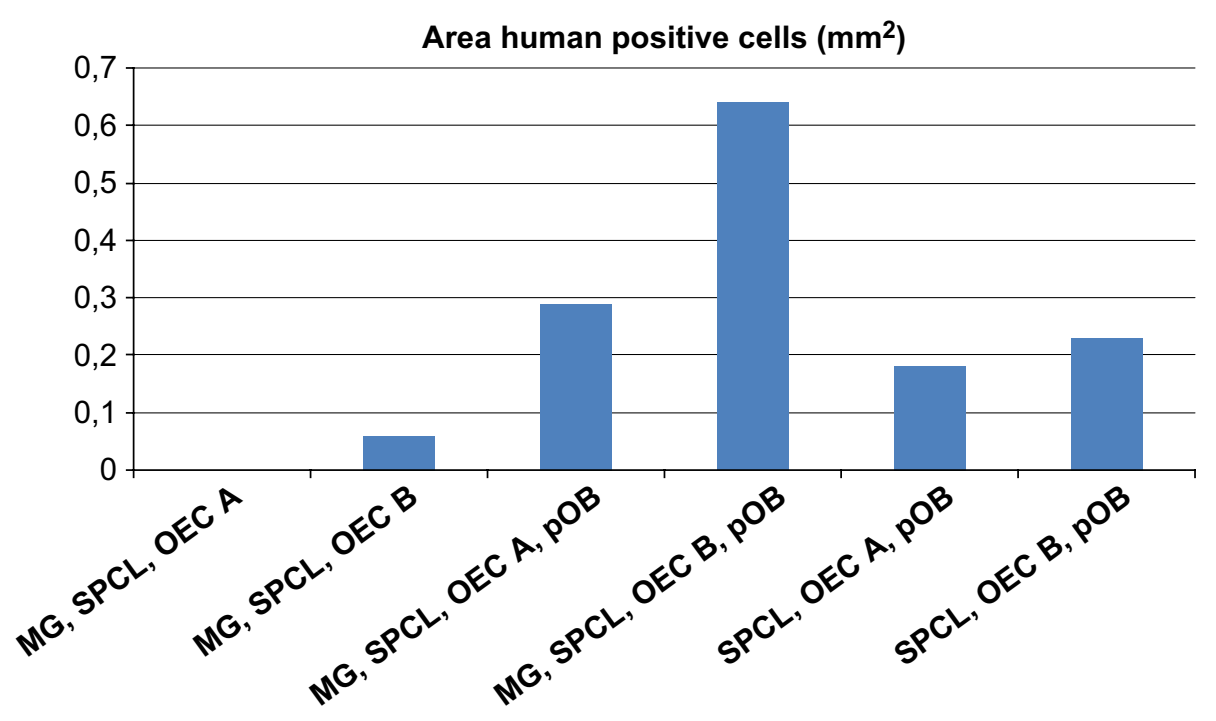

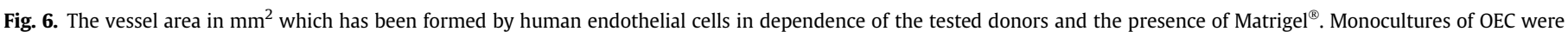
used as additional controls.

determine the effect of the co-culture with $\mathrm{POB}$ on the angiogenic activity of OEC. On the other hand, it is well known that osteogenesis itself is dependent on a functional interaction of endothelial cells and osteoblasts. Initial characterization of complex SPCL scaffolds by quantitative real time PCR indicated the upregulation of the late osteoblastic marker osteocalcin and a decrease in the early marker alkaline phosphatase with progressing pre-culture time (Fig. 3). These data indicate the progressing differentiation of osteoblastic cells in complex SPCL constructs under the culture conditions mainly tailored for OEC. More detailed investigation of the dynamics of endothelial and osteogenic markers, matrix components and vessel formation within complex constructs in vitro is also provided in an additional study based on silk fibroin scaffolds (manuscript submitted), whereas the present paper focuses on the beneficial effect of the co-cultures on the vascularization process by OEC. Nevertheless, the in vivo bone formation of such constructs needs to be investigated in further studies using additional models suitable for monitoring bone formation.

\section{Conclusions}

This manuscript describes the contribution of human OEC, a distinct subpopulation within EPC cultures from the peripheral blood, to the vascularization of a bone tissue construct in a nude mouse model in vivo. Co-cultures or co-implantation of OEC with pOB exerted a beneficial effect on the vascularization process by OEC. The regeneration and repair of highly vascularized tissues and larger tissue defects such as in bone might profit from the use of OEC as autologous cell sources. Our findings might have an impact on further developments of prevascularized tissues for bone based on endothelial progenitor cells.

\section{Acknowledgements}

The authors would like to thank B. Malenica, C. Braun, L. Meyer, K. Molter, M. Müller. J. Alonso-Monje for their excellent technical assistance. This work was financially supported by grants from the European commission (HIPPOCRATES N NMP3-CT-2003-505758; EXPERTISSUES Contract nr.: 500283-2) and BMBF-Grant for German-Chinese Cooperation in Regenerative Medicine (grant number 0315033).

\section{Appendix}

Figures with essential colour discrimination. Certain figures in this article, in particular parts of Figures 1,2,4 and 5 are difficult to interpret in black and white. The full colour images can be found in the on-line version, at doi: 10.1016/j.biomaterials.2008.09.058.

\section{References}

[1] Levenberg S, Rouwkema J, Macdonald M, Garfein ES, Kohane DS, Darland DC, et al. Engineering vascularized skeletal muscle tissue. Nat Biotechnol 2005:23(7):879-84.

[2] Berthod F, Germain L, Tremblay N, Auger FA. Extracellular matrix deposition by fibroblasts is necessary to promote capillary-like tube formation in vitro. J Cell Physiol 2006;207(2):491-8.

[3] Elbjeirami WM, West JL. Angiogenesis-like activity of endothelial cells cocultured with VEGF-producing smooth muscle cells. Tissue Eng 2006.

[4] Saiki A, Watanabe F, Murano T, Miyashita Y, Shirai K. Hepatocyte growth factor secreted by cultured adipocytes promotes tube formation of vascular endothelial cells in vitro; 2006.

[5] Unger RE, Sartoris A, Peters K, Motta A, Migliaresi C, Kunkel M, et al. Tissuelike self-assembly in cocultures of endothelial cells and osteoblasts and the formation of microcapillary-like structures on three-dimensional porous biomaterials. Biomaterials 2007;28(27):3965-76.

[6] Fuchs S, Hofmann A, Kirkpatrick CJ. Microvessel-like structures from outgrowth endothelial cells from human peripheral blood in 2-dimensional and 3-dimensional co-cultures with osteoblastic lineage cells. Tissue Eng 2007;13(10):2577-88.

[7] Wenger A, Stahl A, Weber H, Finkenzeller G, Augustin HG, Stark GB, et al Modulation of in vitro angiogenesis in a three-dimensional spheroidal coculture model for bone tissue engineering. Tissue Eng 2004;10(9-10): $1536-47$.

[8] Villars F, Guillotin B, Amedee T, Dutoya S, Bordenave L, Bareille R, et al. Effect of HUVEC on human osteoprogenitor cell differentiation needs heterotypic gap junction communication. Am J Physiol Cell Physiol 2002;282(4):C775-85.

[9] Spector JA, Mehrara BJ, Greenwald JA, Saadeh PB, Steinbrech DS, Bouletreau PJ et al. Osteoblast expression of vascular endothelial growth factor is modulated by the extracellular microenvironment. Am J Physiol Cell Physiol 2001;280(1): C72-80.

[10] Choong CS, Hutmacher DW, Triffitt JT. Co-culture of bone marrow fibroblasts and endothelial cells on modified polycaprolactone substrates for enhanced potentials in bone tissue engineering. Tissue Eng 2006;12(9):2521-31.

[11] Guillotin B, Bareille R, Bourget C, Bordenave L, Amedee J. Interaction between human umbilical vein endothelial cells and human osteoprogenitors triggers pleiotropic effect that may support osteoblastic function. Bone 2008.

[12] Scherberich A, Galli R, Jaquiery C, Farhadi J, Martin I. Three-dimensional perfusion culture of human adipose tissue-derived endothelial and osteoblastic progenitors generates osteogenic constructs with intrinsic vascularization capacity. Stem Cells 2007;25(7):1823-9.

[13] Rouwkema J, de Boer J, Van Blitterswijk CA. Endothelial cells assemble into a 3-dimensional prevascular network in a bone tissue engineering construct. Tissue Eng 2006;12(9):2685-93. 
[14] Lin Y, Weisdorf DJ, Solovey A, Hebbel RP. Origins of circulating endothelial cells and endothelial outgrowth from blood. J Clin Invest 2000;105(1):71-7.

[15] Gulati R, Jevremovic D, Peterson TE, Chatterjee S, Shah V, Vile RG, et al. Diverse origin and function of cells with endothelial phenotype obtained from adult human blood. Circ Res 2003;93(11):1023-5.

[16] Melero-Martin JM, Khan ZA, Picard A, Wu X, Paruchuri S, Bischoff J. In vivo vasculogenic potential of human blood-derived endothelial progenitor cells. Blood 2007;109(11):4761-8.

[17] Fuchs S, Hermanns M, Kirkpatrick C. Retention of a differentiated endothelial phenotype by outgrowth endothelial cells isolated from human peripheral blood and expanded in long-term cultures. Cell Tissue Res 2006:1-14.

[18] Au P, Daheron LM, Duda DG, Cohen KS, Tyrrell JA, Lanning RM, et al. Differential in vivo potential of endothelial progenitor cells from human umbilical cord blood and adult peripheral blood to form functional long-lasting vessels. Blood 2008;111(3):1302-5.

[19] Au P, Tam J, Fukumura D, Jain RK. Bone marrow-derived mesenchymal stem cells facilitate engineering of long-lasting functional vasculature. Blood 2008;111(9):4551-8

[20] Santos MI, Fuchs S, Gomes ME, Unger RE, Reis RL, Kirkpatrick CJ. Response of micro- and macrovascular endothelial cells to starch-based fiber meshes for bone tissue engineering. Biomaterials 2007;28(2):240-8.

[21] Gomes ME, Holtorf HL, Reis RL, Mikos AG. Influence of the porosity of starchbased fiber mesh scaffolds on the proliferation and osteogenic differentiation of bone marrow stromal cells cultured in a flow perfusion bioreactor. Tissue Eng 2006;12(4):801-9.

[22] Gomes ME, Sikavitsas VI, Behravesh E, Reis RL, Mikos AG. Effect of flow perfusion on the osteogenic differentiation of bone marrow stromal cells cultured on starch-based three-dimensional scaffolds. J Biomed Mater Res A 2003;67(1):87-95.

[23] Fuchs S, Motta A, Migliaresi C, Kirkpatrick CJ. Outgrowth endothelial cells isolated and expanded from human peripheral blood progenitor cells as a potential source of autologous cells for endothelialization of silk fibroin biomaterials. Biomaterials 2006;27(31):5399-408.

[24] Hofmann A, Konrad L, Gotzen L, Printz H, Ramaswamy A, Hofmann C. Bioengineered human bone tissue using autogenous osteoblasts cultured on different biomatrices. J Biomed Mater Res A 2003;67(1):191-9.

[25] Hafemann B, Ensslen S, Erdmann C, Niedballa R, Zuhlke A, Ghofrani K, et al. Use of a collagen/elastin-membrane for the tissue engineering of dermis. Burns 1999;25(5):373-84.

[26] Sanz L, Santos-Valle P, Alonso-Camino V, Salas C, Serrano A, Vicario JL, et al. Long-term in vivo imaging of human angiogenesis: Critical role of bone marrow-derived mesenchymal stem cells for the generation of durable blood vessels. Microvasc Res 2008;75(3):308-14.

[27] Jiang A, Pan W, Milbauer LC, Shyr Y, Hebbel RP. A practical question based on cross-platform microarray data normalization: are BOEC more like large vessel or microvascular endothelial cells or neither of them? J Bioinform Comput Biol 2007;5(4):875-93. 